\title{
Genomic instability and DNA ploidy are linked to DNA copy number aberrations of 8p23 and 22q11.23 in gastric cancers
}

\author{
SHIGETO KAWAUCHI ${ }^{1}$, TOMOKO FURUAY ${ }^{1}$, TETSUJI UCHIYAMA ${ }^{2}$, ATSUSHI ADACHI $^{2}$, TAKAE OKADA ${ }^{1}$ \\ MOTONAO NAKAO ${ }^{1}$, ATSUNORI OGA ${ }^{1}$, KENICHIRO UCHIDA ${ }^{1}$ and KOHSUKE SASAKI ${ }^{1}$ \\ ${ }^{1}$ Department of Pathology, Yamaguchi University Graduate School of Medicine, \\ Ube 755-8505; ${ }^{2}$ Department of Surgery, Iwakuni Medical Center, Iwakuni 740-0021, Japan
}

Received April 8, 2010; Accepted June 2, 2010

DOI: 10.3892/ijmm_00000470

\begin{abstract}
The close relationship between chromosomal instability (CIN) and aneuploidy has been reported. The purpose of this study was to identify genomic aberrations present with CIN and aneuploidy in gastric cancers. FISH and image cytometry were applied to 27 sporadic gastric adenocarcinomas to identify CIN-positive tumors and to determine DNA ploidy, respectively. In addition, array-based comparative genomic hybridization was used to identify bacterial artificial chromosome clones that displayed differences in the frequency of copy number aberrations between CIN-positive and CIN-negative tumors, and between aneuploid and diploid tumors. There were many chromosomal regions with DNA copy number aberrations, some of which were nonrandom aberrations linked to the CIN phenotype and aneuploidy. A copy number loss of 22q11.23 was more frequent in CIN-positive cancers than in others $(7 / 12$ vs. $2 / 15, \mathrm{p}<0.01)$ and in aneuploid cancers than in diploid cancers $(8 / 16$ vs. $1 / 11, p<0.05)$. The frequency of 22q11.23 loss differed significantly between CIN-positive and aneuploid tumors and between CIN-negative and diploid cancers $(7 / 10$ vs. $1 / 9, \mathrm{p}<0.01)$. In contrast, a DNA copy number gain of 8 p23.2 was detected in 6 out of 9 CINnegative/diploid cancers, but was not detected in CINpositive/aneuploid cancers $(\mathrm{p}<0.01)$. There were no cancers carrying both aberrations (22q11.23 loss and 8p23.2 gain). The present study indicates that a 22q11.23 loss and a 8 p23.2 gain are markers for both CIN and aneuploidy. This is the first report describing an inverse relationship between the 22q11.23 loss and 8p23.2 gain in terms of genomic instability and DNA ploidy in gastric cancers.
\end{abstract}

Correspondence to: Dr Kohsuke Sasaki, Department of Pathology, Yamaguchi University Graduate School of Medicine, Ube, Yamaguchi 755-8505, Japan

E-mail: kohsuke@yamaguchi-u.ac.jp

Key words: genomic instability, DNA ploidy, DNA copy number aberration, gastric cancer

\section{Introduction}

Cancer progression is accompanied by the accumulation of genetic alterations in the genes controlling cell proliferation and death. Indeed, some genomic abnormalities, ranging from point mutations to chromosomal aberrations, are detected in virtually all cancers, including gastric cancers. Although the biological characteristics of cancers greatly vary from case to case, they are primarily affected by genomic alterations. In general, cancer cells inherently take on genomic instability that is conceptually divided into microsatellite instability (MIN) and chromosomal instability (CIN) $(1,2)$.

MIN tumors are characterized by replication errors in simple repetitive microsatellite sequences, while CIN tumors are characterized by genetic abnormalities at the chromosome level. Cancers with CIN undergo successive genomic alterations, thus resulting in an increase in malignancy together with clonal selection pressures. Usually, patient prognosis is poorer in CIN-positive tumors than in MIN tumors (2).

CIN is a major source of genomic instability in gastric cancers and accounts for $60 \%$ of these cancers $(4,5)$. It is suggested that CIN develops through a number of different pathways $(3,6,7)$, and more than 100 genes are known to be involved in CIN $(3,8)$. However, it is presently uncertain whether certain genomic alterations are linked to CIN in gastric cancers. Aneuploidy and genomic instability have long been recognized as one of the hallmarks of cancer $(1-3,6)$. CIN causes an imbalance in chromosome numbers (aneuploidy), which is considered to be a surrogate marker of CIN $(1,2)$.

The relationship between genomic instability and DNA ploidy is well recognized; MIN and CIN are closely linked to diploid and aneuploid cancers, respectively $(1,6)$. The frequency of aneuploidy is approximately $60 \%$ in gastric cancers $(5,9,10)$. Aneuploidy results from a number of causes including mitotic defects such as asymmetrical mitoses, tetraploidy, and centrosomal abnormalities (6). Aneuploidy and CIN are inextricably linked $(1,5,11)$. This suggests that the genes implicated in the development of aneuploidy play a concurrent role in the development of CIN. The identification of recurrent genomic abnormalities common to CIN and DNA aneuploidy offer insight into the mechanisms of DNA aneuploidy and CIN.

The technology of array-based $\mathrm{CGH}(\mathrm{aCGH})$ is a powerful tool for identifying the chromosomal regions with 
DNA copy number aberrations in tumors. In this study, we identified the genes linked with both genomic instability and DNA ploidy in gastric adenocarcinoma by means of aCGH technology. A copy number loss of 22q11.23 was preferentially detected in CIN-positive aneuploid cancers, while a 8p23.2 copy number gain was present exclusively in CIN-negative diploid cancers. There were no tumors that simultaneously exhibited an 8p23.2 gain and 22q11.23 loss. These observations therefore support the hypothesis that genomic instability and DNA ploidy are closely linked.

\section{Materials and methods}

Tissue specimens and DNA samples. Twenty-seven consecutive surgically resected gastric adenocarcinomas were used, including 2 early cancers and 25 advanced cancers. The patients consisted of 16 males and 11 females ranging from 46 to 88 years of age with an average age of 71.1. The family histories were non-contributing factors for all patients and all tumors were considered to be sporadic. The clinicopathological features are summarized in Table I. In brief, according to Lauren's histological classification, 14 tumors were classified as intestinal-type gastric cancers, while the remaining tumors were diffuse-type cancers. Node metastasis was detected in 19 cancers. Tissue specimens were stored at $-80^{\circ} \mathrm{C}$ until use. A tissue microdissection technique was applied to $10-\mu \mathrm{m}$ frozen tissue sections in order to reduce the contamination of normal tissue components for aCGH analyses as previously described (12).

In addition, touch smears were prepared by touching thawed tissue specimens to glass slides after wiping blood from the cut surface of the specimens with a paper towel as described previously $(4,5,11)$. One touch sample was fixed with $70 \%$ ethanol for DNA measurement by laser scanning cytometry (LSC) $(5,13,14)$. The others were dried and fixed with $100 \%$ ethanol for FISH analysis (5,11-15). The study protocol was conducted under the approval of the Institutional Review Board for human tissue use at the Yamaguchi University School of Medicine in 2004, and informed consent for this study was obtained from every patient.

LSC. DNA ploidy was determined as described previously $(5,13,14)$. Touch preparations fixed in $70 \%$ ethanol were dipped in PI solution $(25 \mu \mathrm{g} / \mathrm{ml}$ in PBS) containing $0.1 \%$ RNase (Sigma-Aldrich, St. Louis, MO, USA). A coverslip was placed on the slide and sealed with nail polish. The DNA content was measured with a laser scanning cytometer (LSC 101, Olympus Co., Tokyo, Japan). Approximately 5000 cells were examined per sample. DNA histograms were generated, and DNA ploidy was determined. A DNA index (DI) of 1.0 indicates DNA diploidy, and tumors with $1.0<\mathrm{DI}<1.2$ were classified as near-diploid cases and were included in a DNA diploid group. Cancers with $\mathrm{DI} \geq 1.2$ were classified as DNA aneuploid tumors.

FISH. Touch smears fixed in $100 \%$ ethanol were refixed in $0.2 \%$ paraformaldehyde-PBS at $4^{\circ} \mathrm{C}$ for $10 \mathrm{~min}$ as previously described $(5,11,13-15)$. We examined the numerical aberrations in chromosomes $7,11,17$, and 18

Table I. Clinicopathological summary of 27 gastric adenocarcinomas.
Total number of gastric
cancers examined
Average age of patients (years)
Range of age of patients (years)
Gender (F/M)
Histological type of gastric cancers
Intestinal-type
Diffuse-type

Node metastasis

Liver metastasis

Peritoneal dissemination

Early cancers ${ }^{\mathrm{a}}$

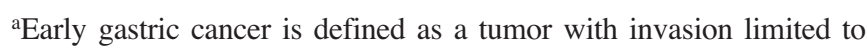
the mucosa or submucosa according to the Japanese Classification of

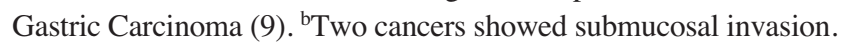

using SpectrumGreen or SpectrumRed labeled alphoid satellite DNA probes specific for the pericentromeric region of each chromosome (D7Z1, D11Z1, D17Z1, and D18Z1; Abbott Molecular Inc., Des Plaines, IL), as previously described $(5,13-15)$. In brief, $3 \mu 1$ of the probe mixture was applied to the touch preparations, covered with a coverslip, and denatured at $73^{\circ} \mathrm{C}$ for $2 \mathrm{~min}$. The slides were incubated overnight at $39^{\circ} \mathrm{C}$ and washed at $73^{\circ} \mathrm{C}$. The slides were then transferred to a solution of $2 \mathrm{X} \mathrm{SSC} / 0.1 \% \mathrm{NP}-40$ at room temperature. DNA was stained with DAPI-II (4,6-diamidino2-phenylindole-II, Vysis), and a coverslip was placed on the slide.

The number of hybridization signals in each nucleus was determined by observing more than 200 nuclei on each slide with a fluorescence microscope equipped with a x100 oil immersion objective lens (Olympus Co.). The percentages of cells with different signal counts were calculated for each slide. The degree of intercellular numerical variation for each chromosome was defined as the fraction of cells whose chromosome number differed from the modal chromosome number $(5,11,14)$.

Statistical analysis of FISH and LSC results. The Student's t-test was used to compare the population size of tumor cells with signal spots equivalent to the modal chromosome number between two tumor groups (diploid versus aneuploid tumor specimens) in gastric cancers based on DNA indices. Statistical significance was set at $\mathrm{p}<0.05$.

$a C G H$. High molecular weight genomic DNA was extracted from microdissected tumor tissue specimens with a DNA extraction kit (SepaGene, Sankojunyaku Co., Ltd, Tokyo, Japan) as previously described $(13,16)$. Control DNA (Promega, Madison, WI) was used as a reference. Array 
Table II. The relationship between ploidy and CIN in 27 gastric cancers.

CIN-positive CIN-negative No. of tumors

\begin{tabular}{lrrr}
\hline Diploid & 2 & 9 & 11 \\
Aneuploid & 10 & 6 & 16 \\
No. of tumors & 12 & 15 & 27 \\
\hline
\end{tabular}

CGH experiments were performed with MacArray Karyo 4000 (Macrogen Inc., Seoul, Korea) according to the manufacturer's protocol. The arrays consisted of 4,030 human bacterial artificial chromosome (BAC) clones spotted in triplicate, spanning the entire human genome at an average interval of $1 \mathrm{Mb}$ (Macrogen Inc., Rockville, MD). Sampleand gender-matched reference genomic DNAs (500 ng each) were labeled using the random priming method with fluorescence dyes Cy 3 and Cy 5, respectively. The labeled DNAs were mixed with Cot-1 DNA (50 mg, Gibco BRL, Gaithersburg, MD) and were then hybridized to the array slides for 2 days at $37^{\circ} \mathrm{C}$ in a humidified chamber. The array slides were rinsed in a washing buffer and dried well. The array slides were scanned with a GenePix 4000A scanner (Axon Instruments, Union City, CA). The fluorescence images were analyzed using the Mac Viewer software program (Macrogen, Inc.) optimized for the analysis of the array as previously reported (17-19). Fluorescence spots were defined with the automatic grid feature and adjusted manually. All $\mathrm{CGH}$ ratios were converted to log base 2 . The ratios of the fluorescence intensities of all the spots were plotted against the distance of the clones along the chromosomes. For each BAC clone, average ratios that deviated significantly from zero were considered to be abnormal $\left( \pm \log _{2} 0.25\right)$.

Analysis of aCGH data. The clonal frequency of DNA copy number aberrations (DCNAs) between the two groups, diploid and aneuploid cancers, and between CIN-positive and -negative cancers were each compared using a $\chi^{2}$ test to identify the BAC clones that were used for distinguishing between the two groups. The Fisher's exact test was applied for the statistical analysis of the difference in the frequency of specific changes between two groups. A difference was considered significant at $\mathrm{p}<0.05$.

\section{Results}

LSC and DNA ploidy. All tumors, including aneuploid tumors, showed a distinct diploid peak in a DNA histogram. Seven tumors had a single diploid peak, and 4 tumors had an additional peak $(1.0<\mathrm{DI}<1.2)$ near the diploid peak. In total, 11 tumors were classified as diploid. Sixteen tumors showed a distinct peak (DI>1.2) in addition to the diploid peak (Table II). DIs ranged from 1.0 to 2.64 in this series.

FISH and CIN. In normal mucosae, $>80 \%$ of cells had 2 signal spots for all the chromosomes that were examined: On average, the percentage $( \pm \mathrm{SD})$ of disomy was $87.7 \pm 4.4$,
$87.4 \pm 4.4,84.9 \pm 5.6$ and $86.8 \pm 6.2 \%$ for chromosomes 7,11 , 17 and 18 , respectively. No polysomic ( $>3$ signals) cells were observed. In gastric cancers, the chromosome copy number varied across tumors. In diploid tumors, disomic cells were predominant for all chromosomes examined, while in aneuploid tumors, trisomic or tetrasomic cells were frequently present. When the percentage of cells with a modal chromosome number of $<70 \%$ and if $>2$ chromosomes adopted this criterion, the tumor was defined as a CIN-positive cancer. In this series, 12 and 15 were classified into CIN-positive and CIN-negative cancers, respectively (Table II).

DNA ploidy and CIN. Ten out of 12 (83.3\%) CIN-positive cancers were aneuploid, and 9 out of $15(60.0 \%)$ CIN-negative cancers were diploid. Alternatively, CIN features were detected in 10 out of $16(62.5 \%)$ aneuploid tumors and 2 out of $11(18.2 \%)$ diploid tumors. Six out of 27 (22.2\%) gastric cancers were classified as CIN-negative/aneuploid cancers, and $2(7.4 \%)$ as CIN-positive/diploid cancers (Table II).

$a C G H$. A DNA copy number gain was frequent in chromosomes 7, 8 and 20, and in particular, a gain of 8q24.13 was detected in $77 \%$ of gastric cancers. A DNA copy number loss was frequent in chromosomal regions Yp11, 8p23 and 14q32, and a loss of Yp11.2 was detected in $67 \%$ of the cancers (Fig. 1).

DCNAs vs. DNA ploidy and CIN. There are many chromosomal regions with copy number aberrations, of which some are nonrandom phenomena linked to CIN phenotype and aneuploidy. A clone-by-clone comparison of the frequency of DCNAs between diploid and aneuploid gastric cancers revealed BAC clones (chromosomal regions) that displayed significant differences in the frequency of DCNAs between these two groups (Table III). Gains of Xp28, 8q24.3, 8p23.3 and 8 p23.1 were more frequent in diploid tumors than in aneuploid tumors. In contrast, gains of 5 p15.2 and $6 \mathrm{p} 12.1$, 5q14.2, 9p24.3, 9p24.3, 19p13.13, and losses of 19p13.11 were not detected in diploid tumors.

Several BAC clones showed different frequencies of DCNAs between CIN-positive and -negative cancers (Table IV). A gain of 1p31.2 was detected exclusively in CIN-positive tumors (7/12). In contrast, a gain of $8 \mathrm{q} 22.3$ was detected in CIN-negative cancers (9/15), but not in CINpositive cancers. Gains of 5p15.33, 6p24.3 and 8p23.3 and losses of $15 q 26.3$ and $4 q 12$ were exclusively detected in CIN-positive cancers.

The gain of 8p23.2 and loss of 22q11.23 were linked to both DNA ploidy and genetic instability. A gain of 8p23.2 was detected in 7 (25.9\%) gastric cancers. All of the cancers with an 8 p23.2 gain were CIN-negative $(\mathrm{p}<0.01)$, and 6 $(85.7 \%)$ of these were diploid $(\mathrm{p}<0.01)$. On the other hand, an 8p23.2 gain was detected in $7(46.7 \%)$ of CIN-negative cancers, and $6(54.5 \%)$ of these were diploid tumors (Fig. 2). A gain of 8 p23 was detected in only $1(6.2 \%)$ aneuploid tumor, but was not detected in CIN-positive cancers. A gain of 8 p23.2 was detected in $6(66.7 \%)$ out of 9 CINnegative/diploid cancers, but was not detected in any of the 10 CIN-positive/aneuploid cancers $(p<0.01)$ (Fig. 2). A BAC clone corresponding to the chromosomal region 8 p23.2 
Table III. Difference in the frequency of DCNAs between diploid and aneuploid gastric cancers.

\begin{tabular}{|c|c|c|c|c|}
\hline \multirow[b]{2}{*}{ Region } & \multirow[b]{2}{*}{ Gene(s) } & \multirow[b]{2}{*}{ p-value } & \multicolumn{2}{|c|}{ Frequency } \\
\hline & & & Diploid & Aneuploid \\
\hline \multicolumn{5}{|l|}{ Gain } \\
\hline $\mathrm{Xq} 28$ & $\begin{array}{l}\text { CSAG3A, CSAG2, MAGEA12, CSAG1, } \\
\text { MAGEA2, MAGEA2B, MAGEA3 }\end{array}$ & 0.001333502 & $7 / 11$ & $1 / 16$ \\
\hline $8 \mathrm{q} 24.3$ & $\begin{array}{l}\text { BOP1, HSF1, DGAT1, SCRT1, FBXL6, } \\
\text { GPR172A, ADCK5 }\end{array}$ & 0.002811979 & $5 / 11$ & $0 / 16$ \\
\hline $8 \mathrm{p} 23.3$ & ERICH1 & 0.004897228 & $6 / 11$ & $1 / 16$ \\
\hline $8 \mathrm{p} 23.1$ & AGPAT5 & 0.005035697 & $8 / 11$ & $3 / 16$ \\
\hline $5 \mathrm{p} 15.2$ & & 0.005179172 & $0 / 11$ & $8 / 16$ \\
\hline $6 \mathrm{p} 12.1$ & HMGCLL1 & 0.005179172 & $0 / 11$ & $8 / 16$ \\
\hline $8 \mathrm{q} 24.3$ & $\begin{array}{l}\text { CYHR1, KIFC2, FOXH1, PPP1R16A, GPT, } \\
\text { MFSD3, RECQL4, LRRC14, LRRC24 }\end{array}$ & 0.005613232 & $7 / 11$ & $2 / 16$ \\
\hline \multicolumn{5}{|l|}{ Loss } \\
\hline $5 q 14.2$ & RPS23 & 0.010806427 & $0 / 11$ & $7 / 16$ \\
\hline $9 \mathrm{p} 24.3$ & DOCK8 & 0.010806427 & $0 / 11$ & $7 / 16$ \\
\hline $9 \mathrm{p} 24.3$ & DMRT3, DMRT2 & 0.010806427 & $0 / 11$ & $7 / 16$ \\
\hline $19 \mathrm{p} 13.13$ & RTBDN, MAST1, DNASE2, KLF1, GCDH, FARSLA & 0.010806427 & $0 / 11$ & $7 / 16$ \\
\hline $19 \mathrm{p} 13.11$ & RAB3A, PDE4C, KIAA1683, JUND & 0.010806427 & $0 / 11$ & $7 / 16$ \\
\hline $\begin{array}{l}19 \mathrm{p} 13.3 \\
22 \mathrm{q} 11.22\end{array}$ & PPAP2C, MIER2, THEG & 0.012655237 & $1 / 11$ & $9 / 16$ \\
\hline$-22 q 11.23$ & RTDR1, GNAZ, RN7SLP3 RAB36, BCR & 0.012655237 & $1 / 11$ & $9 / 16$ \\
\hline $22 q 11.23$ & C22orf15, C22orf16, MMP11, SMARCB1, DERL3 & 0.019143341 & $1 / 11$ & $8 / 15$ \\
\hline
\end{tabular}

Table IV. Difference in the frequency of DCNAs between gastric and CIN-negative and -positive gastric cancers.

\begin{tabular}{|c|c|c|c|c|}
\hline \multirow[b]{2}{*}{ Region } & \multirow[b]{2}{*}{ Candidate gene(s) } & \multirow[b]{2}{*}{$\mathrm{p}$-value } & \multicolumn{2}{|c|}{ Frequency } \\
\hline & & & CIN (-) & CIN (+) \\
\hline \multicolumn{5}{|l|}{ Gain } \\
\hline $1 \mathrm{q} 31.2$ & & 0.000588344 & $0 / 15$ & $7 / 12$ \\
\hline $8 \mathrm{q} 22.3$ & & 0.001487434 & $9 / 15$ & $0 / 11$ \\
\hline $5 p 15.33$ & SDHA & 0.005613232 & $0 / 15$ & $5 / 12$ \\
\hline $6 \mathrm{p} 24.3$ & BMP6 & 0.005613232 & $0 / 15$ & $5 / 12$ \\
\hline $8 \mathrm{p} 23.3$ & ERICH1 & 0.005967800 & $0 / 15$ & $7 / 12$ \\
\hline \multicolumn{5}{|l|}{ Loss } \\
\hline $15 q 26.3$ & CHSY1 & 0.001900893 & $0 / 15$ & $6 / 12$ \\
\hline $4 q 12$ & C4orf14, POLR2B, IGFBP7 & 0.005613232 & $0 / 15$ & $5 / 12$ \\
\hline $21 q 22.11$ & ITSN1, ATP5O & 0.005967800 & $7 / 15$ & $0 / 12$ \\
\hline $22 q 11.23$ & C22orf15, C22orf16, MMP11, SMARCB1, DERL3 & 0.007729806 & $2 / 15$ & $7 / 11$ \\
\hline $22 q 11.21$ & GGT2 & 0.009374769 & $5 / 15$ & $10 / 12$ \\
\hline
\end{tabular}

(BAC clone \#5831) harbors ERICH1 (glutamate-rich 1). In contrast, loss of 22q11.23 was detected in $9(33.3 \%)$ gastric cancers, and was more frequent in aneuploid and/or CIN- positive cancers than in diploid and/or CIN-negative cancers, respectively $(\mathrm{p}<0.05$ and $\mathrm{p}<0.01$, respectively). Of these 9 cancers with a 22q11.23 loss, 7 were CIN-positive and 


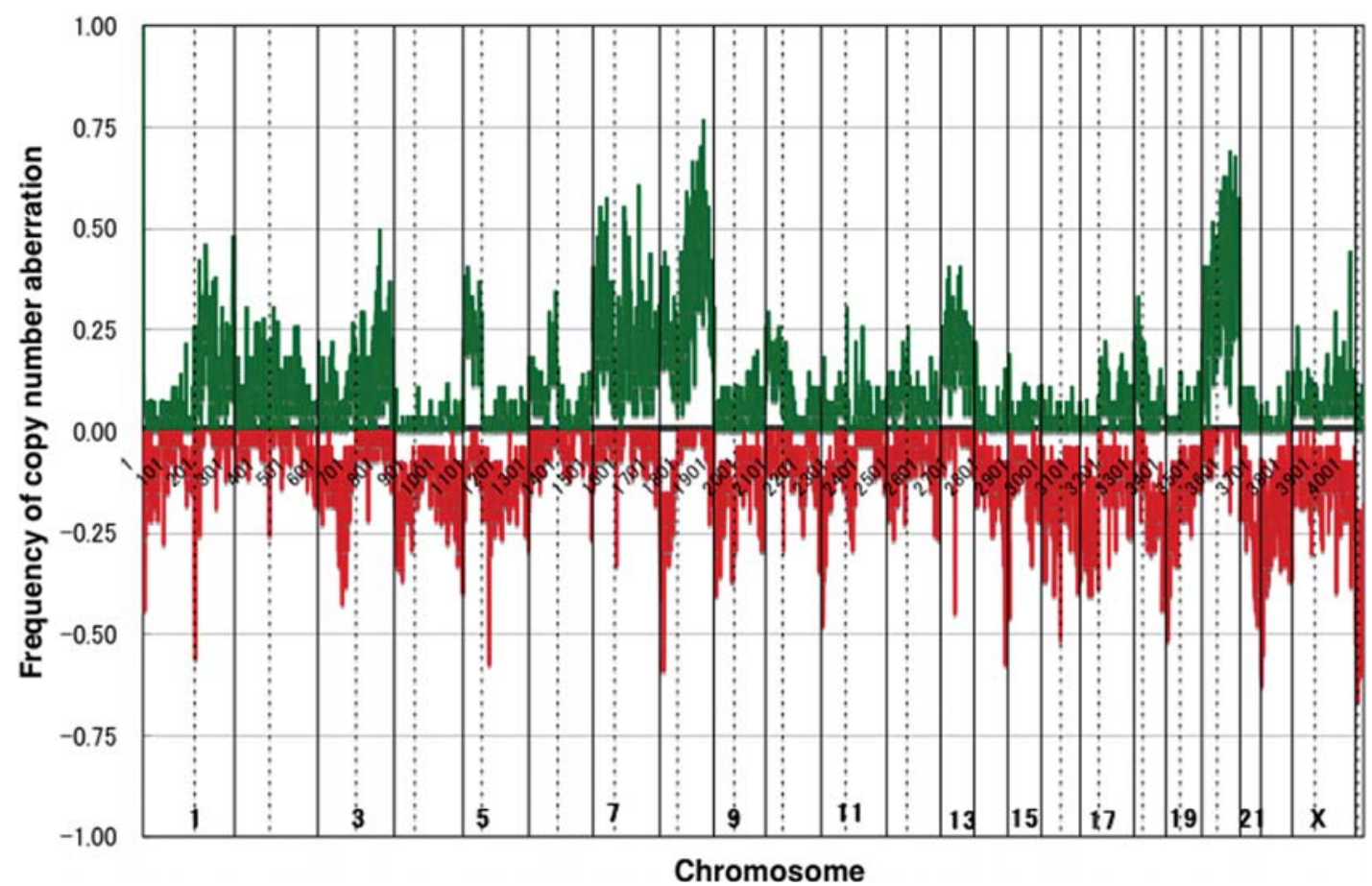

Figure. 1. The frequency of copy number aberrations for each bacterial artificial chromosome (BAC) clone in 27 gastric adenocarcinomas. DNA copy number aberrations were detected by array-based CGH using an array spotted with 4,030 human BAC clones that spanned the whole human genome at an average interval of $1 \mathrm{Mb}$. A DNA copy number gain was frequent in chromosomes 7 and 8, and a gain of 8q24.13 was detected in $77 \%$ of 27 gastric cancers (green line). A DNA copy number loss was frequent in chromosomal regions Yp11,8p23 and 14q32, and a loss of Yp11.2 was detected in 67\% of gastric cancers (red line). The upper part of the figure shows the frequency of DNA copy number aberrations, and the lower part depicts the frequency of DNA copy number losses. The ordinate axis indicates the frequency of copy number aberrations for each BAC clone, and the abscissa axis shows the chromosomal regions corresponding to the BAC clones.

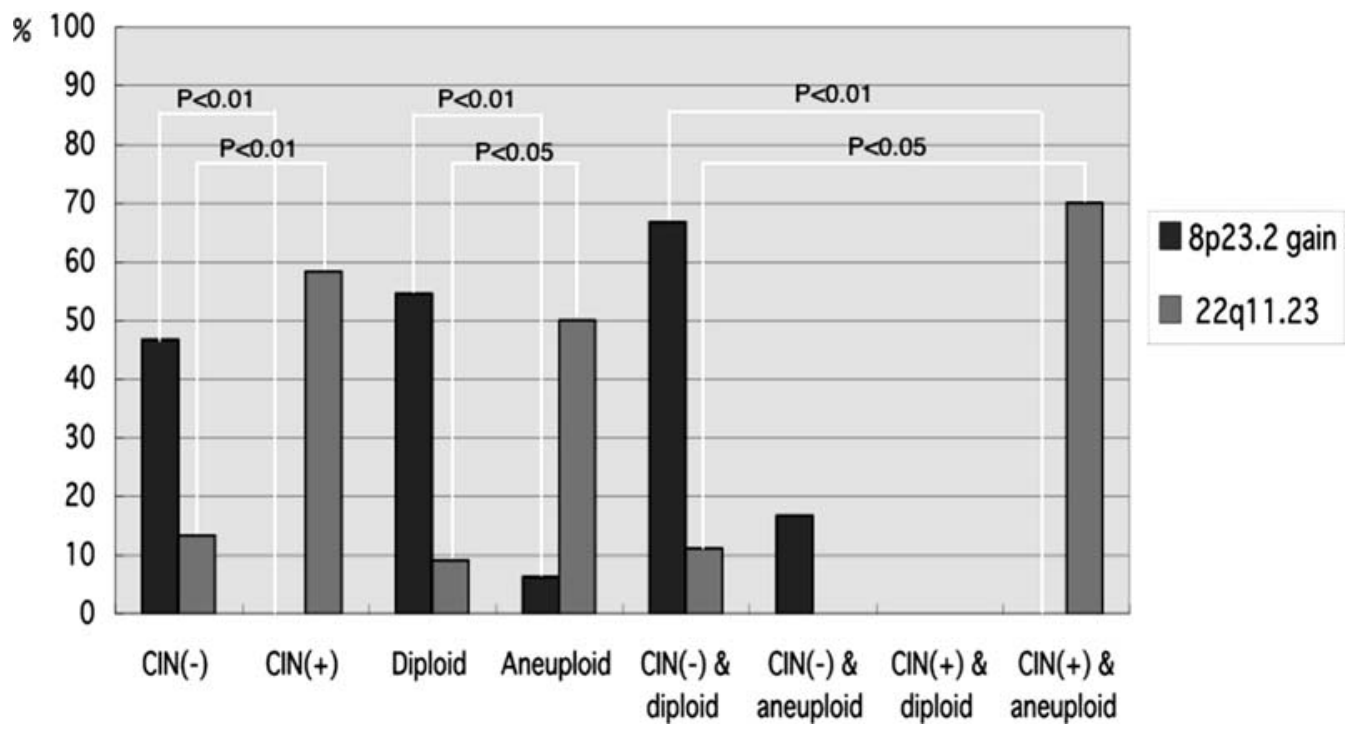

Figure. 2. Comparison of the frequency of a 8p23.2 gain and 22q11.23 loss between diploid and aneuploid tumors, and CIN-negative and -positive cancers. An 8p23.2 gain was detected in 54.4 and $6.3 \%$ of diploid and aneuploid tumors, respectively, and in 46.7 and $0 \%$ of CIN-negative and -positive cancers, respectively. The frequency of an 8p23.2 gain is significantly different between diploid and aneuploid tumors (p<0.01), and between CIN-negative and -positive cancers (p<0.01). A 22q11.23 loss is detected in 9.1 and 50.0\% of diploid and aneuploid tumors, respectively, and in 13.3 and $58.3 \%$ of CIN-negative and -positive cancers, respectively. The frequency of a 22q11.23 loss is significantly different between diploid and aneuploid tumors (p<0.05), and between CIN-negative and -positive cancers $(\mathrm{p}<0.01)$. In addition, an 8 p23.2 gain and 22q11.23 loss are detected in 66.7 and $11.1 \%$ of CIN-negative diploid cancers, respectively, while they are detected in 0 and $70 \%$ of CIN-positive aneuploid cancers, respectively. The frequency of an 8 p23.2 gain and 22q11.23 loss was significantly different between CIN-negative diploid and CIN-positive aneuploid cancers ( $\mathrm{p}<0.01$ and $\mathrm{p}<0.05$, respectively).

aneuploid tumors. One of the remaining 2 tumors displayed CIN-negative and diploid features, and the other showed CIN-negative and aneuploid features. Loss of 22q11.23 was detected in 7 out of $10(70 \%)$ CIN-positive/aneuploid cancers, but was only detected in one CIN-negative/diploid tumor $(\mathrm{p}<0.05)$. There were no tumors with a $22 \mathrm{q} 11.23$ gain. 
The BAC clone for 22q11.23 (clone \#2661) harbors genes such as MMP11, SMARCB1 and DERL3.

There were no tumors that simultaneously exhibited an 8p23.2 gain and 22q11.23 loss, although 11 (40.7\%) tumors displayed neither of these chromosomal abnormalities.

\section{Discussion}

The differentiation between CIN-positive and -negative cancers is biologically important. Twelve out of 27 (44.4\%) gastric cancers were classified as CIN-positive cancers. The percentage of CIN-positive cancers observed in this series of tumors is consistent with reports by others $(5,20,21)$. Although $\mathrm{CIN}$ and aneuploidy are not equivalent, they are interrelated in gastric cancers. In the present study, $83.3 \%$ of CIN-positive cancers were aneuploid, and $62.5 \%$ of aneuploid tumors were CIN-positive. For convenience, aneuploidy is considered a surrogate marker of CIN in tumors $(1,2,6)$. These facts suggest that the development of CIN and aneuploidy share common genetic pathways. In this context, the identification of genomic abnormalities common to both CIN and aneuploidy may help elucidate the molecular interrelationship between CIN and aneuploidy in tumors. Many mechanisms that maintain genomic stability are known, and several genes involved in genomic instability have been identified $(3,6,7)$. However, genomic aberrations common to CIN and the development of aneuploidy have not been reported in gastric cancers or other tumors.

The comparison of aCGH data between diploid and aneuploid tumors and between CIN-positive and -negative cancers revealed that a gain of 8 p23.2 and a loss of $22 \mathrm{q} 11.23$ correlated with both ploidy and genomic stability. An 8p23.2 gain was preferentially detected in diploid or CIN-negative cancers, but was not detected in CIN-positive cancers. This suggests a close relationship between the gain of gene(s) at 8p23.2 and ploidy and chromosomal stability. The BAC clone corresponding to the chromosomal region 8p23.2 contains no oncogenes or tumor suppressor genes, but does contain the ERICH1 gene. However, there are no reports on the relationship of a gain in the ERICH1 expression to ploidy and genomic stability. This genomic change may have an inhibitory effect against the development of CIN and aneuploidy in gastric cancer, although it is difficult to validate the mechanisms whereby an 8p23.2 gain is linked to diploidy or CIN-negativity.

Conversely, a loss of 22q11.23 was preferentially detected in aneuploid or CIN-positive cancers, and was detected in nearly $70 \%$ of CIN-positive/aneuploid cancers. A BAC clone corresponding to chromosomal region $22 \mathrm{q} 11.23$ bears several genes including SMARCB1, which is a tumor suppressor gene (22). It is likely that a loss of $22 \mathrm{q} 11.23$ induces a loss of SMARCB1. SMARCB1 inactivation is present in malignant rhabdoid tumors and epithelioid sarcomas (22-24). However, the relationship between SMARCB 1 loss and CIN and aneuploidy has not been previously reported. We cannot exclude the possibility that a loss of $22 \mathrm{q} 11.23$ is a prerequisite for both CIN and aneuploidy in gastric cancers, because in general the loss of tumor suppressor function leads to CIN and aneuploidy (25). Regardless of whether 22q11.23 copy number aberrations are causal or correlative of the CIN phenotype, the mechanism by which a 22q11.23 loss contributes to aneuploidy and CIN remains to be investigated. CIN and aneuploidy may be induced by factors other than a $22 \mathrm{q} 11.23$ loss, as not all cancers with a 22q11.23 loss exhibited CIN and aneuploidy.

It is significant that no gastric cancers simultaneously had both an 8p23.2 gain and 22q11.23 loss. An 8p23.2 gain and $22 q 11.23$ loss were inversely correlated with genomic instability and DNA ploidy: A 22q11.23 loss is a marker of CIN-positive or aneuploid cancers, while an 8 p23.2 gain is a marker of CIN-negative or diploid tumors. Therefore, these changes facilitate the genomic classification of gastric cancers, which differs from the conventional histological classification. Cells have a system for maintaining ploidy and genomic stability. This suggests that the same relationship between these genomic changes and genomic instability may therefore be present in other tumors as well as in gastric cancers.

\section{Acknowledgements}

This study was supported in part by the Ministry of Education, Culture, Sports, Science, and Technology of Japan (grant no. 21390106) and The New Energy and Industrial Technology Development Organization (NEDO) of Japan (grant no. 21659088).

\section{References}

1. Lengauer C, Kinzler KW and Vogelstein B: Genetic instability in colorectal cancers. Nature 386: 623-627, 1997.

2. Lengauer C, Kinzler KW and Vogelstein B: Genetic instabilities in human cancers. Nature 396: 643-649, 1998.

3. Jefford CE and Irminger-Finger I: Mechanisms of chromosome instability in cancers. Crit Rev Oncol Hematol 59: 1-14, 2006.

4. Choi SW, Park SW, Lee KY, et al: Fractional allelic loss in gastric carcinoma correlates with growth patterns. Oncogene 17: 2655-2659, 1998.

5. Furuya T, Uchiyama T, Murakami T, et al: Relationship between chromosomal instability and intratumoral regional DNA ploidy heterogeneity in primary gastric cancers. Clin Cancer Res 6: 2815-2820, 2000.

6. Rajagopalan H and Lengauer C: Aneuploidy and cancer. Nature 432: 338-341, 2004.

7. Gagos S and Irminger-Finger I: Chromosome instability in neoplasia: chaotic roots to continuous growth. Int J Biochem Cell Biol 37: 1014-1033, 2005.

8. Michor F: Chromosomal instability and human cancer. Philos Trans R Soc Lond B Biol Sci 29: 631-635, 2005.

9. Furuya T, Uchiyama T, Adachi A, et al: Relation of DNA ploidy to genetic aberrations detected by chromosomal CGH and FISH in gastric adenocarcinomas. Oncol Rep 15: 1491-1496, 2006.

10. Lee KH, Lee JS and Suh C: DNA flow cytometry of stomach cancer. Cancer 72: 1819-1826, 1993.

11. Miyazaki M, Furuya T, Shiraki A, et al: The relationship of DNA ploidy to chromosomal instability in primary human colorectal cancers. Cancer Res 59: 5283-5285, 1999.

12. Hashimoto Y, Oga A, Okami K, Imate Y, Yamashita Y and Sasaki K: Relationship between cytogenetic aberrations by CGH coupled with tissue microdissection and DNA ploidy by laser scanning cytometry in head and neck squamous cell carcinoma. Cytometry 40: 161-166, 2000.

13. Yamamoto Y, Matsuyama H, Furuya T, et al: Centrosome hyperamplification predicts progression and tumor recurrence in bladder cancer. Clin Cancer Res 10: 6449-6455, 2004.

14. Yamamoto Y, Matsuyama H, Kawauchi S, et al: Biological characteristics in bladder cancer depend on the type of genetic instability. Clin Cancer Res 12: 2752-2758, 2006.

15. Ikemoto K, Furuya T, Matsuda K, et al: Multicolor FISH and cytometric analyses allow classification of urothelial carcinomas into two subtypes, low- and high-grade tumors. Int J Oncol 25: 893-898, 2004. 
16. Noutomi Y, Oga A, Uchida K, et al: Comparative genomic hybridization reveals genetic progression of oral squamous cell carcinoma from dysplasia via two different tumourigenic pathways. J Pathol 210: 67-74, 2006.

17. Furuya T, Uchiyama T, Adachi A, et al: The development of a mini-array for estimating the disease states of gastric adenocarcinoma by array CGH. BMC Cancer 8: 393, 2008.

18. Chochi Y, Kawauchi S, Nakao M, et al: A copy number gain of the $6 \mathrm{p}$ arm is linked with advanced hepatocellular carcinoma. J Pathol 217: 677-684, 2009.

19. Tsuji K, Kawauchi S, Saito S, et al: Breast cancer cell lines carry cell line-specific genomic alterations that are distinct from aberrations in breast cancer tissues: Comparison of the $\mathrm{CGH}$ profiles between cancer cell lines and primary cancer tissues. BMC Cancer 10: 15, 2010.

20. Ottini L, Falchetti M, Lupi R, et al: Patterns of genomic instability in gastric cancer: clinical implications and perspectives. Ann Oncol 17: 97-102, 2006.

21. Hiyama T, Tanaka S, Yoshihara M, et al: Chromosomal and microsatellite instability in sporadic gastric cancer. J Gastroenterol Hepatol 19: 756-760, 2004.
22. Isakoff MS, Sansam CG, Tamayo P, et al: Inactivation of the Snf5 tumor suppressor stimulates cell cycle progression and cooperates with p53 loss in oncogenic transformation. Proc Natl Acad Sci USA 102: 17745-17750, 2005.

23. Sigauke E, Rakheja D, Maddox DL, et al: Absence of expression of SMARCB1/INI1 in malignant rhabdoid tumors of the central nervous system, kidneys and soft tissue: an immunohistochemical study with implications for diagnosis. Mod Pathol 19: 717-725, 2006.

24. Modena P, Lualdi E, Facchinetti F, et al: SMARCB1/INI1 tumor suppressor gene is frequently inactivated in epithelioid sarcomas. Cancer Res 65: 4012-4019, 2005.

25. Shen KC, Heng H, Wang Y, et al: ATM and p21 cooperate to suppress aneuploidy and subsequent tumor development. Cancer Res 65: 8747-8753, 2005. 